\title{
Spububaúde
}

\section{Abdome agudo obstrutivo por íleo biliar}

\author{
Acute obstructive abdome by ileo biliar
}

\section{Bruna Santana Novais $^{1}$, Beatriz Carvalho De Campos Bueno ${ }^{2} \bullet$, Francisca Dias De Moraes $^{3} \bullet$, Lorena Murta Dos Santos ${ }^{4} \bullet$, Felipe Augusto Ferreira Siquelli ${ }^{5} \bullet$, Rafaela Santos $\operatorname{Costa}^{6}{ }^{\circ}$, Ted Renato Marçal Fumagalli ${ }^{7} \bullet$, Marcelo Jacques Segal ${ }^{8^{*}} \odot$}

\begin{abstract}
${ }^{1}$ Graduada em Medicina na Pontifícia Universidade Católica, Campinas, São Paulo, Brasil. ${ }^{2}$ Graduanda em Medicina na Pontifícia Universidade Católica, Campinas, São Paulo, Brasil. ${ }^{3}$ Graduanda em Medicina na Pontifícia Universidade Católica, Campinas, São Paulo, Brasil. ${ }^{4}$ Graduanda em Medicina na Pontifícia Universidade Católica, Campinas, São Paulo, Brasil. ${ }^{5}$ Médico residente em Cirurgia Geral do

Hospital da Pontifícia Universidade Católica de Campinas, Campinas, São Paulo, Brasil. ${ }^{6}$ Médico residente no hospital da Pontifícia

Universidade Católica, Campinas, São Paulo, Brasil. ${ }^{7}$ Médico residente no hospital da Pontifícia Universidade Católica, Campinas, São Paulo, Brasil. ${ }^{8}$ Médico assistente no hospital da Pontifícia Universidade Católica, Campinas, São Paulo, Brasil. *Autor para correspondência.

E-mail: mevsegal@ig.com.br
\end{abstract}

\begin{abstract}
Resumo: Introdução: A doença calculosa biliar pode ter como rara complicação a formação de fístula entre a vesícula biliar (VB) e o intestino, com migração de cálculo biliar para a luz intestinal sendo responsável por entre 1 a $4 \%$ dos quadros de abdome agudo obstrutivo. Relato de caso: Apresentamos uma paciente portadora de colelitíase, cuja VB fístulizou-se com o duodeno, gerando um abdome agudo obstrutivo devido cálculo biliar impactado no intestino delgado, a aproximadamente $15 \mathrm{~cm}$ da válvula ileocecal (VIC). Submetida à laparotomia exporadora com enterotomia do segmento e retirado o cálculo. Discussão: Apesar de íleo biliar configurar uma condição rara, seu diagnóstico e tratamento precoces são imprescindíveis para que haja menor morbidade e mortalidade nesses pacientes os quais são idosos em sua maioria.
\end{abstract}

Palavras-chave: obstrução intestinal, colelitíase, enterolitotomia, colecistite, íleo biliar.

\begin{abstract}
Introduction: A rare complication such as gallstone disease occurs through the fistulas formation between the gallbladder and the intestine, with migration of the gallstone to the intestinal lumen, responsible for 1 to $4 \%$ of cases of acute obstructive abdomen. Case report: We present a patient with cholelithiasis, whose gallbladder fistulates in the duodenum, generating acute obstructive abdomen due to impacted gallstone in the small intestine, approximately $15 \mathrm{~cm}$ from the ileocecal valve. Undergo a laparotomy with segmental enterotomy and the stone removed. Discussion: Although gallstone ileus is a rare condition, its early diagnosis and treatment are essential for to be less morbidity and mortality in these patients, mostly elderly.
\end{abstract}

Keywords: abdomen, acute, biliary fistula, cholecystitis, gallbladder diseases.

\section{Introdução}

O íleo biliar é uma condição rara que afeta principalmente mulheres e idosos com história prévia de colelitíase, representando 0,3 a $0,5 \%$ das complicações biliares e 1 a $4 \%$ das causas de obstrução intestinal (Turner et al., 2021).

O primeiro diagnóstico se deu por Bartholin em 1654 ao identificar um cálculo biliar ocasionando obstrução na topografia de jejuno. Após o caso pioneiro, diversos outros foram relatados na literatura (Martin, 1912).

Sua fisiopatologia é precedida da formação de uma fístula colecistoentérica, que pode causar obstrução intestinal quando o(s) cálculo(s) ganha(m) a luz intestinal, migrando distalmente e impactando em regiões de menor diâmetro ao longo do trato digestivo. Os sítios de obstrução mais comuns são, em ordem decrescente de prevalência: íleo (50 a 60,5\%), jejuno (16,1-26,9\%) e duodeno (3,5 a 14,6\%) (Reisner \& Cohen, 1994; Inukai, 2019). Os cálculos geralmente são compostos por colesterol (Beuran et al., 2010), apresentando controvérsias na literatura quanto às dimensões, com registros variando dentre 0,5 a $5,0 \mathrm{~cm}$, com a moda em $3 \mathrm{~cm}$ (Javanthi et al., 2001) e publicações com notas de sua maioria apresentando diâmetro superior a $2,5 \mathrm{~cm}$ (Beuran et al., 2010).

A suspeita diagnóstica é aventada a partir de uma Radiografia simples de abdome cujos achados consistem de dilatação de alças de delgado com nível hidroaéreo, litíase biliar ectópica e pneumobilia, contemplando a tríade de Rigler, patognomônica da doença, presente entre 40 e 50\% dos casos (Inukai, 2019; Towndend et al., 
2012; Javanthi et al., 2001). Se os achados radiográficos não forem sugestivos e se ainda houver suspeita clínica, a Tomografia computadorizada (TC) de abdome está indicada, com sensibilidade diagnóstica de 93\% (Clavien et al., 1990).

O diagnóstico cirúrgico diferencial pode ainda incluir Apendicite, Diverticulite, Síndrome de Mirizzi e a Síndrome de Bouveret (Alemi et al., 2019).

O tratamento do Íleo Biliar é cirúrgico sendo individualizado de acordo com o sítio de obstrução do cálculo e o fechamento da fístula, podendo ser alcançado através de cirurgia em um ou dois tempos (Turner et al., 2021; Inukai, 2019).

A taxa de mortalidade situa-se entre 12 e 17\%, devido principalmente à dificuldade do diagnóstico e à epidemiologia da doença, que afeta frequentemente idosos com comorbidades diversas (Reisner \& Cohen, 1994).

\section{Relato de Caso}

A divulgação deste trabalho foi autorizada pelo pesquisador responsável Dr. Marcelo Jacques Segal. Paciente do sexo feminino, 90 anos, natural de Pernambuco, procedente de Campinas, viúva, dois filhos, sem profissão declarada, hipertensa, hipotireoideia, dá entrada no Hospital - PUC Campinas com queixa de vômitos e diarréia liquida, sem sangue ou muco, após ingestão de alimento supostamente estragado no mesmo dia.

Ao exame físico inicial mostrava-se hipocorada, desidratada $++/ 4$, anictérica, acianótica e afebril. O abdome encontrava-se flácido, com ruídos hidroaéreos (RHA) aumentados, doloroso à palpação em região epigástrica, sem sinais de peritonite, com Raio X (RX) de abdome agudo evidenciando distensão de delgado.

Inicialmente tratada como quadro de intoxicação alimentar, mas apresentou refratariedade dos sintomas com exarcebação do quadro de náuseas e vômitos além de dor abdominal inespecífica e mais intensa no mesogástro. O exame físico abdominal evoluiu com distensão, hipertimpanismo e palpação dolorosa do mesogastro, ausência de massas palpáveis ou visceromegalias, descompressão brusca e sinal de Murphy negativos.

Por suspeita de abdome agudo obstrutivo, realizou-se TC de abdome sem contraste por função renal alterada, revelando distensão líquida de alças intestinais no mesogastro, cálculo com centro radioluscente medindo 2,2 x 1,5 cm impactado no íleo distal, distando $25 \mathrm{~cm}$ da Válvula Íleo Cecal (VIC), determinando moderada distensão de alças intestinais à montante inclusive em câmara gástrica. Vesícula Biliar (VB) com paredes espessadas, focos gasosos em seu interior e aparente trajeto fistuloso com o duodeno, achados compatíveis com quadro de oclusão intestinal por cálculo biliar (Figuras 1A e 1B).
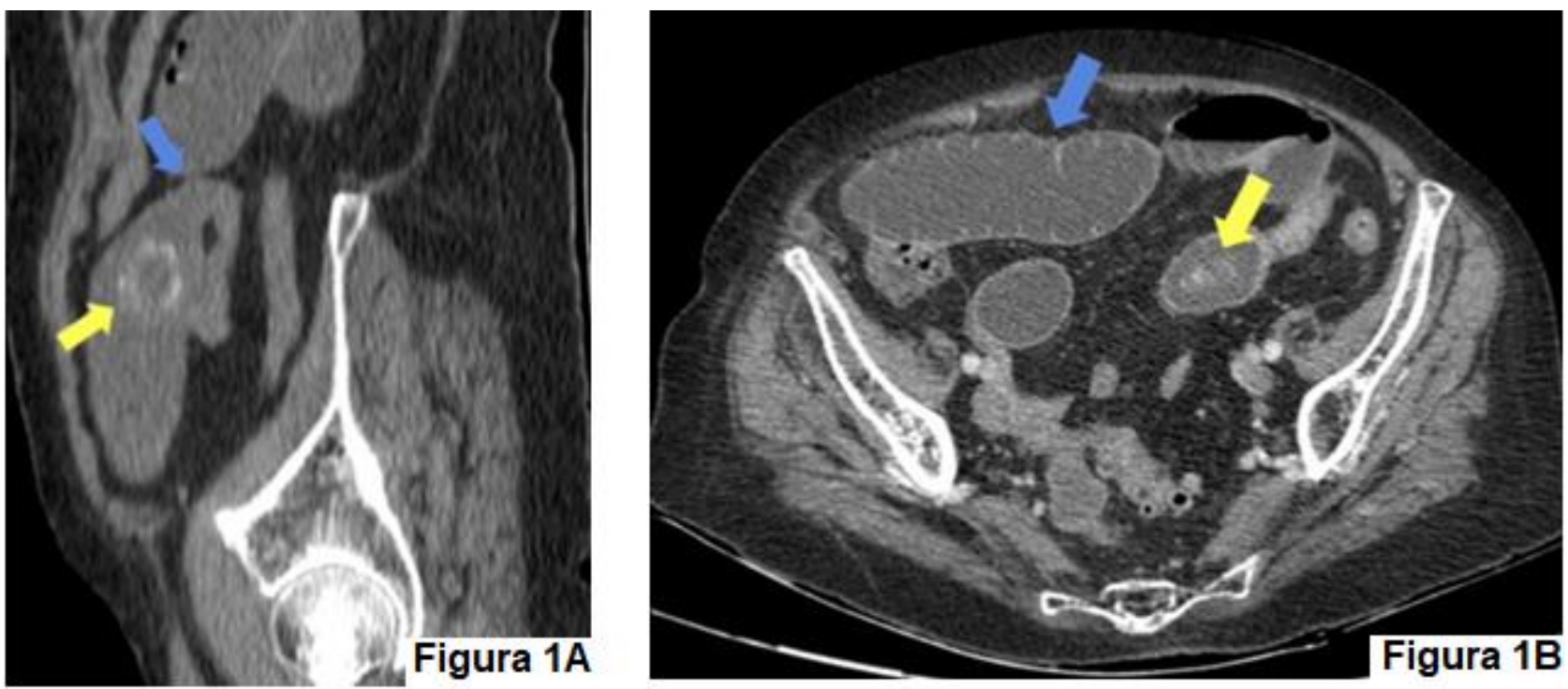

Figura 1. (A) Corte sagital. Seta amarela - presença de cálculo em luz intestinal com centro radioluscente e bordas radiopacas. Seta azul - Ponto de afilamento abrupto do calibre da alça intestinal logo após o cálculo. (B) Corte axial. Seta amarela - cálculo em luz intestinal. Seta azul - Distensão de alças.

A paciente foi submetida à laparotomia exploradora por abdome agudo obstrutivo devido íleo biliar. No inventário da cavidade foi evidenciado alças de delgado distendidas à montante à partir de $15 \mathrm{~cm}$ da VIC, sendo a topografia da impactação do cálculo que obstruiu totalmente a luz do intestino (Figuras 2A, 2B e 2C). 
Optado por realizar enterolitotomia, seguida de enterorrafia. A paciente apresentou boa evolução pósoperatória, recebendo alta no terceiro dia de pós-operatório e encaminhada para seguimento ambulatorial.
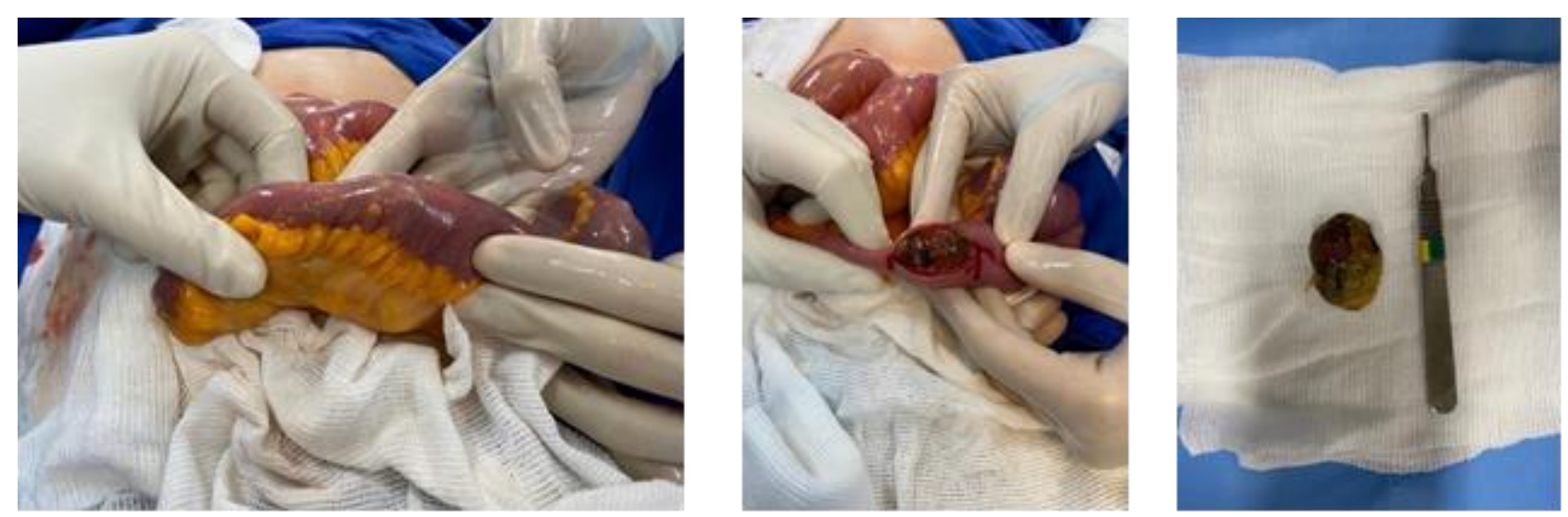

Figura 2. (A) Dilatação de alças a montante e afilamento a jusante, mostrando ponto de impactação do cálculo. (B) Incisão no ponto de impactação do cálculo. (C) Comparação proporcional entre o cálculo e o cabo do bisturi.

\section{Discussão}

O íleo biliar é uma complicação rara de colelitíase. A literatura relata que as mulheres são mais acometidas que os homens, em uma proporção de até 15 vezes maior (Javanthi et al., 2001; Clavien et al., 1990), sendo compatível com o caso clínico.

A sintomatologia clássica do íleo biliar é de vômitos, náuseas e dor abdominal, que são atenuadas conforme a movimentação do cálculo e, caso esse cálculo se impacte novamente, é esperado o retorno dos sintomas. Alguns sintomas que também podem estar presentes são: desidratação, aumento dos RHA e distensão abdominal (Pena et al., 2020).

Embora raro, o íleo biliar pode ser diagnosticado em até $77 \%$ dos casos a partir da história clínica do paciente e exames de imagem, sendo a TC e RX de abdome os exames necessários para tal (Reisner \& Cohen, 1994; Clavien et al., 1990). O tratamento cirúrgico tem como objetivo principal tratar o problema agudo: o íleo biliar. A fístula poderá ser abordada no mesmo tempo cirúrgico, a depender da idade e comorbidades do paciente, gravidade da oclusão e se há outros achados locais. Dessa forma os procedimentos cirúrgicos serão enterolitotomia, exploração da fístula (podendo ser abordado também o ducto colédoco) e colecistectomia (Inukai, 2019).

Outro dado extremamente relevante quando se discute a abordagem do íleo biliar é entender, conforme descrição literária, qual o perfil do paciente acometido. De maioria idosa e normalmente com comorbidades associadas (Abich et al., 2017), a execução do planejamento cirúrgico (isto é, enterolitotomia, colecistectomia e abordagem da fístula) pode oferecer um risco relevante de mortalidade de até $25 \%$ dos casos (Reisner \& Cohen, 1994). Portanto, cabe uma análise sobre a abordagem cirúrgica em duas etapas, sendo realizada na primeira a enterolitotomia e na segunda a colecistectomia, abordagem da fístula e do ducto colédoco conforme necessidade (Redaelli et al., 1997). É recomendado que seja feita a antibioticoprofilaxia visto que a principal complicação do procedimento é a infecção da ferida operatória (Reisner \& Cohen, 1994).

\section{Considerações finais}

O diagnóstico é realizado em mais de $75 \%$ dos casos a partir da história e exames de imagens complementares, a maioria dos casos de íleo biliar só será diagnosticada após laparotomia exploradora devido a um abdome agudo obstrutivo, pois essa conclusão dificilmente é colocada como hipótese diagnóstica, seja pela inespecificidade dos sintomas e dos achados laboratoriais ou pela dificuldade de se pensar no diagnóstico.

Portanto, esse relato de caso visa trazer à comunidade médica um exemplo de como o íleo biliar pode se manifestar, expor as dificuldades de conclusão diagnóstica, bem como apontar o caminho para o tratamento da urgência cirúrgica. Apesar de ser um diagnóstico conhecido de longa data, a hipótese diagnóstica é dificultada pela inespecificidade dos achados, ficando o íleo biliar como diagnóstico de exclusão. 


\section{Referências}

Abich E., Glotzer D., \& Murphy. E. 2017. Gallstone Ileus: An Unlikely Cause of Mechanical Small Bowel Obstruction. Case Reports in Gastroenterology, 11(2), 389-395.

Alemi, F., Seiser, N., Ayloo, S. 2019. Gallstone disease - Cholecystitis, Mirizzi Syndrome, Bouveret Syndrome, Gallstona Ileus. The Surgical Clinics North America, 99(2), 231-244.

Beuran, M., Ivanov, I., \& Venter, M. D. 2010. Gallstone ileus: Clinical and Therapeutic aspects. Journal of Medicine and Life, 3(4), 365-371.

Clavien, P. A., Richon, J., Burgan, S., \& Rohner, A. 1990. Gallstone ileus. The British Journal of Surgery, 77(7), $737-42$.

Inukai, K. 2019. Gallstone ileus: a review. BMJ Open Gastroenterology, 6, e000344.

Jayanthi, S. K., Martin, M. G. M., Menezes, M. R., De barros N., \& Cerri, G. G. 2001. Qual o seu diagnóstico. Radiologia Brasileira, 34(3), VII-VIII.

Martin, F. 1912. Intestinal obstruction due to gall-stones: with report of three successful cases. Annals of Surgery, 55(5), 725-74.

Pena, J. H. W., Viana, F. C. T., Faria, J. R. V., Pena, G. S. W., \& Pena, A. C. 2020. Íleo Biliar: Dois Relatos de Caso de Obstrução Intestinal por Cálculo Biliar. Brazilian Jounal of Surgery and Clinical Research - BJSCR, 33.

Redaelli, C. A., Büchler, M. W., Schilling, M. K., Krähenbühl, L., Ruchti, C., Blumgart, L. H., \& Baer, H. U. 1997. High coincidence of Mirizzi syndrome and gallbladder carcinoma. Surgery, 121(1), 58-63.

Reisner, R. M, \& Cohen, J. R. 1994. Gallstone ileus: a review of 1001 reported cases. The American Surgeon, 60(6), 441-446.

Towndend, C. M., Evers, B. M., Mattox, K. L. 2012. Sabiston tratado de cirurgia: a base biológica da moderna prática cirúrgica. 19a ed. Rio de Janeiro: Elsevier, p. 1259-83.

Turner, A. R., Sharma, B., Mukherjee, S. 2021. Gallstone Ileus. Treasure Island (FL): StatPearls Publishing.

\section{Minicurrículo}

Bruna Santana Novais. Possui graduação em Medicina na Pontifícia Universidade Católica de Campinas (PUCCAMP).

Beatriz Carvalho De Campos Bueno. Graduanda em Medicina na Pontifícia Universidade Católica de Campinas (PUCCAMP).

Francisca Dias De Moraes. Graduanda em Medicina na Pontifícia Universidade Católica de Campinas (PUCCAMP).

Lorena Murta Dos Santos. Graduanda em Medicina na Pontifícia Universidade Católica de Campinas (PUCCAMP).

Felipe Augusto Ferreira Siquelli. Médico residente em Cirurgia Geral do Hospital da Pontifícia Universidade Católica de Campinas.

Rafaela Santos Costa. Médica Residente em Cirurgia Geral do Hospital da Pontifícia Universidade Católica de Campinas (PUCCAMP). 
Ted Renato Marçal Fumagalli. Médico Residente em Cirurgia Geral do Hospital da Pontifícia Universidade Católica de Campinas (PUCCAMP).

Marcelo Jacques Segal. Mestre em Ciências Saúde e Médico assistente do Serviço de Cirurgia de Urgência e Trauma (SCUT) do Hospital da Pontifícia Universidade Católica de Campinas (PUCCAMP).

Como citar: Novais, B.S., Bueno, B.C.C., Moraes, F.D., Santos, L.M., Siquelli, F.A.F., Costa, R.S., Fumagalli, T.R.M., \& Segal, M.J. 2021. Abdome agudo obstrutivo por ileo biliar. Pubsaúde, 6, a135. DOI: https://dx.doi.org/10.31533/pubsaude6.a135

Recebido: 17 jan. 2021.

Revisado e aceito: 1 mar. 2021.

Conflito de interesse: os autores declaram, em relação aos produtos e companhias descritos nesse artigo, não ter interesses associativos, comerciais, de propriedade ou financeiros que representem conflito de interesse.

Licenciamento: Este artigo é publicado na modalidade Acesso Aberto sob a licença Creative Commons Atribuição 4.0 (CC-BY 4.0). 